\title{
Constrains about oil and gas exploration in the southern portion of Parana Basin: the Torres Sinclinal Structure
}

Silvia Beatriz Alves Rolim ${ }^{1}$, Daniel Auler ${ }^{1}$, Patricia de Lugão²

${ }^{1}$ UFRGS - Geociências; STRATAIMAGE ${ }^{2}$

Copyright 2016, SBGf - Sociedade Brasileira de Geofísica

Este texto foi preparado para a apresentação no VII Simpósio Brasileiro de Geofísica, Ouro Preto, 25 a 27 de outubro de 2016. Seu conteúdo foi revisado pelo Comitê Técnico do VII SimBGf, mas não necessariamente representa a opinião da SBGf ou de seus associados. É proibida a reprodução total ou parcial deste material para propósitos comerciais sem prévia autorização da SBGt.

\section{Abstract}

The magnetotelluric method (MT) is relatively new in Brazil. However, its credibility is increasing and its use has being intensified. It is able to acquire resistivity data of rocks that are even $20 \mathrm{Km}$ deep, without losing much resolution. This is possible because it operates in frequencies between 1 and $0,0001 \mathrm{~Hz}$ and uses the Earth's natural electromagnetic field as the source of energy. This method is especially effective to acquire data in areas covered with a volcanic layer. This is the case of Torres's Syncline, a region covered by the Parana-Etendeka volcanic province, where massive lava flows cover great sedimentary deposits of Mesozoic/Paleozoic age. This area has potential to unconventional hydrocarbon reservoirs, and the MT is a great method to search potential structural settings for its occurrence.

\section{Introdução}

Atualmente, as reservas de óleo e gás em xisto vem sendo reavaliadas em função da expectativa de produção energética mundial. Os Estados Unidos possuem as maiores reservas de óleo, ao passo que a China possui as maiores reservas de gás em xisto. O Brasil possui a segunda maior reserva de óleo e gás em xisto da América do Sul, com a Argentina ocupando o primeiro lugar. Porém, atualmente, apenas quatro países no mundo extraem essas reservas, com liderança absoluta dos Estados Unidos, seguido do Canadá, China e Argentina. O uso de métodos geofísicos é fundamental na caracterização indireta de rochas e estruturas associadas ao acúmulo de óleo e gás. Além disso, apresentam inúmeras vantagens na obtenção de dados em subsuperfície, principalmente pela gama de informações específicas de forma rápida e baixo custo. Dentre os métodos geofísicos, o Magneto-Telúrico (MT) é pouco utilizado no Brasil. Porém seu uso tem-se mostrado extremamente útil e de crescente credibilidade nos últimos anos, como alternativa aos métodos sísmicos (Philipp et al 2014). Este trabalho discute o arcabouço estrutural da Sinclinal de Torres, na porção Norte do Estado do Rio Grande do Sul, e suas implicações na avaliação do potencial para reservatórios não convencionais. Esta discussão é realizada no contexto estratigráfico do sudeste da Bacia do Paraná, com base na análise 


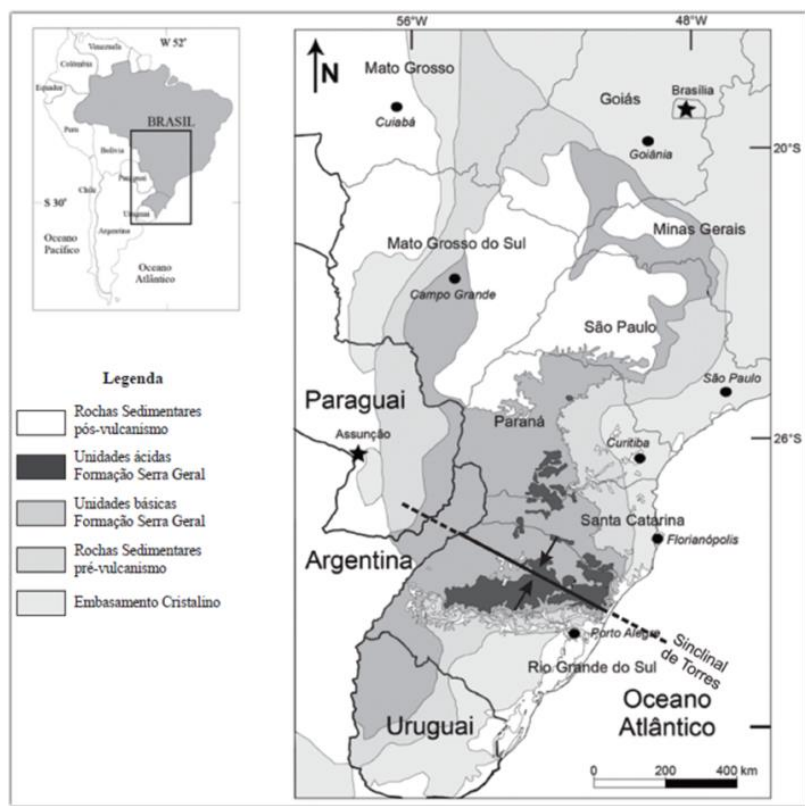

Figura 1 - Mapa geológico da Bacia do Paraná com enfoque na Sinclinal de Torres (Renner, 2010).

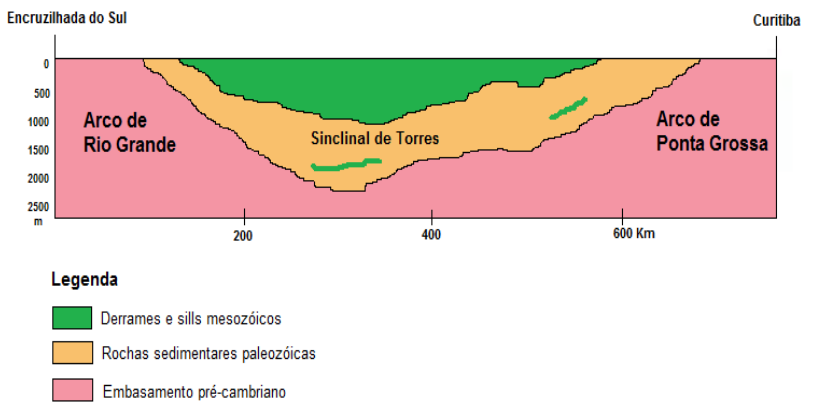

Figura 2 - Seção geológica para a Sinclinal de Torres (Modificado de Soares et al. 2012).

\section{Metodologia/ Problema Investigado}

O método Magnetotelúrico (MT) opera no domínio da frequência, cuja fonte é a variação do campo magnético terrestre, com frequências que variam de $16 \mathrm{kHz}$ a $0,001 \mathrm{~Hz}$. Em um levantamento terrestre, mede-se duas componentes do campo elétrico e três componentes do campo magnético. Os canais elétricos ou telúricos (Ex e Ey) são medidos usando dipolos de 50 a 100m em configuração cruzada, conectados a eletrodos de cloreto de chumbo. As componentes do campo magnético ( $\mathrm{Hx}$, Hy e $\mathrm{Hz}$ ) são medidas por meio de bobinas de indução de alta sensibilidade. As componentes Ex e Hx são alinhadas com o Norte verdadeiro ou com o Norte magnético com a ajuda de uma bússola (figura 1).

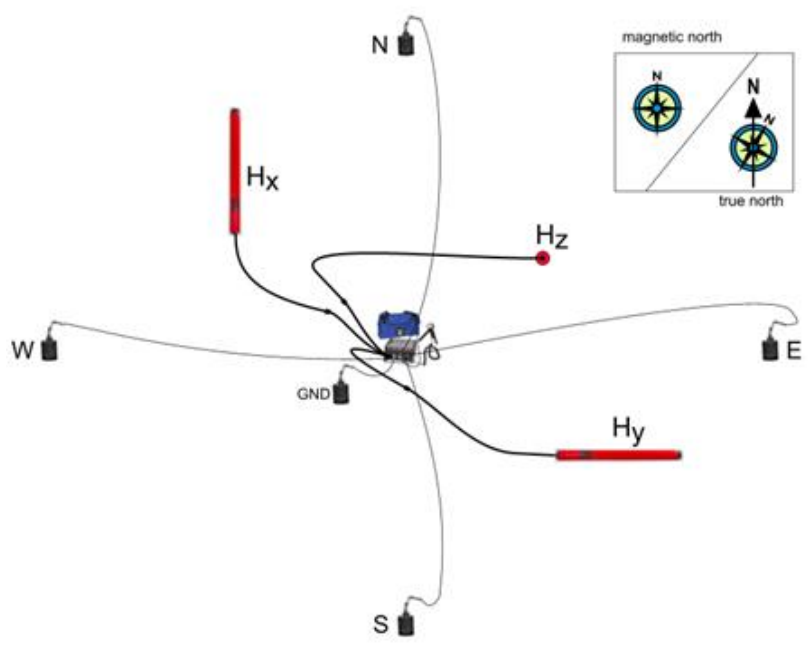

Figura 3 - Disposição do equipamento em campo.

A aquisição de dados pelo método MT envolveu a obtenção de três seções regionais com extensões de 520 km (PSI), 270 km (PSII) e 355 km (PSIII) (Figura 2). Foram utilizados dois (2) equipamentos de medidas magnetotelúrica de banda larga, modelo MTU-5A, fabricado pela Phoenix Geophysics do Canadá O espaçamento entre as estações foram definidos em função da profundidade estimada do embasamento e do grau de detalhamento a ser investigado em cada área. A modelagem foi realizada a partir do Perfil PSI traçado da cidade de Aceguá (RS) até a cidade de Barracão (SC), com o trecho correspondente à Sinclinal de Torres espaçado em $10 \mathrm{~km}$ entre as estações (Figura 2). Os dados MT foram processados utilizando programas próprios do fabricante (Phoenix), resultando em curvas de resistividade aparente e fase, tipper, entre outros parâmetros (Lugão e Rolim, 2012). A modelagem 2D inversa foi executada a partir de modelos preliminares homogêneos. Os parâmetros da inversão são as resistividades dentro de células em uma malha $2 \mathrm{D}$ que se entende lateralmente e em profundidade para considerar condições de contorno e a topografia. Dados de testemunhos de sondagem e mapas geológicos auxiliaram na modelagem.

\section{Resultados}

A modelagem através do método Magneto-Telúrico obteve êxito em termos de resolução e na diferenciação rochas com diferentes valores de resistividade. Para a Sinclinal de Torres, o perfil PSI ofereceu as informações sobre arcabouço geológico-estrutural da região. Os dados da modelagem $2 \mathrm{D}$ comparados com dados obtidos através de testemunhos de sondagem e mapas geológicos. 


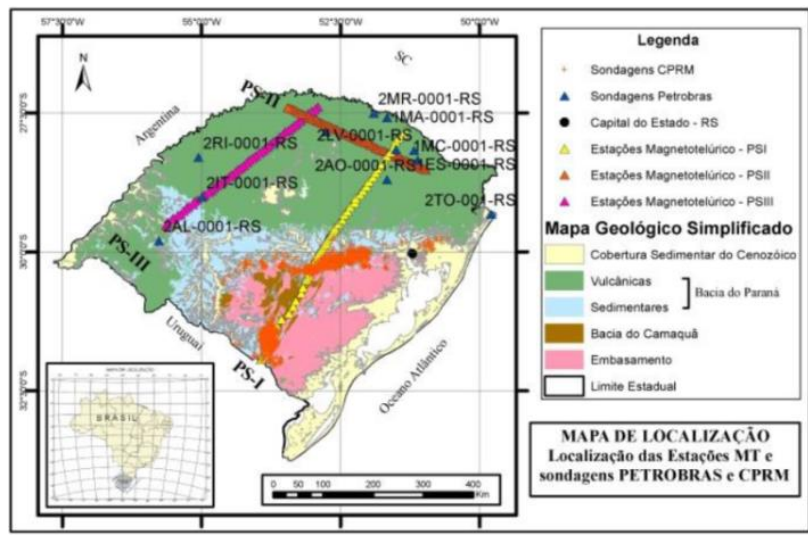

Figura 4 - Localização das transectas em relação ao mapa geológico do Rio Grande do Sul. Na porção SE encontrase a PSI apresentada neste trabalho.

A modelagem 2D dos dados MT mostrou valores de alta resistividade para cobertura vulcânica da Fm. Serra Geral e baixos valores de resistividade associados às rochas sedimentares da Bacia do Paraná. O embasamento apresentou valores de resistividade variando de alta a muito alta (Figura 5 em anexo).

Além dos limites entre os principais pacotes litológicos, também foi possível inferir os principais sistemas de falhas na região, caracterizados por destacadas divergências ou curvas abruptas de sinal, sugerindo deslocamento litológico. As maiores discordâncias estão relacionadas a sistemas do tipo Horst e Grabens. Na sua maioria, as falhas mostram disposição subvertical com rejeito de algumas centenas de metros. A seção PSI que foi utilizada para a interpretação principal da região da Sinclinal de Torres evidencia, principalmente, as falhas de direção NW-SE. Em direção ao norte do perfil PSI é possível detectar um grande aumento na espessura da camada sedimentar da Bacia do Paraná, porção que pertence à borda sul da Sinclinal de Torres. A continuidade destas camadas é interrompida por sistemas de falhas regionais, destacando-se as falhas Santo Ângelo - Barra do Ribeiro, Não Me Toque - Capão da Canoa e Torres - Posadas (Figura 5 em anexo).

\section{Discussão e Conclusões}

O uso de métodos geofísicos é fundamental na caracterização indireta de rochas e estruturas associadas ao acúmulo de óleo e gás. A caracterização tectônica de terrenos Mesozóicos e Paleozóicos é fundamental na investigação de estruturação de ambientes com potencial para geração de reservatórios não convencionais de rochas vulcânicas em bacias sedimentares com potencial de geração de hidrocarbonetos. O Magneto-Telúrico (MT) é uma alternativa aos métodos sísmicos, principalmente pela grande quantidade de informações estruturais e litológicas de subsuperfície recuperadas de forma rápida e baixo custo. Neste trabalho, o método MT permitiu a caracterização de unidades abaixo da cobertura vulcânica da Formação Serra Geral, diferente dos métodos sísmicos que trabalham com ondas mecânicas. Desta forma, o arcabouço estrutural da Sinclinal de Torres foi definido na porção Norte do Estado do Rio Grande do Sul, auxiliando a reavaliação do potencial para reservatórios não convencionais. Os resultados da modelagem 2D de um perfil MT transversal à Sinclinal de Torres reforçaram as evidências de uma calha NW-SE profunda na porção norte do Estado do Rio Grande do Sul. Dados offshore de levantamentos sísmicos. A seção PSI que foi utilizada para a interpretação principal da região da Sinclinal de Torres evidencia, principalmente, as falhas de direção NW-SE. Em direção ao norte do perfil PSI é possível detectar um grande aumento na espessura da camada sedimentar da Bacia do Paraná, porção que pertence à borda sul da Sinclinal de Torres.

\section{Agradecimentos}

Os autores agradecem ao Laboratório de Sensoriamento Remoto Geológico da UFRGS e às empresas Petrobrás e Strata Image que viabilizaram a execução deste trabalho de pesquisa.

\section{Referências}

Bologna, M. S.; Nunes, H. O.; Padilha, A. L.; Vitorello, I.; Padua, M. B., 2013. Anomalous electrical structure in the northwestern Parana Basin, Brazil, observed with broadband magnetotellurics. Journal of South American Earth Sciences; v. 42, p. 74-82, MAR 2013

Colombo, D.; Keho, T.; Janoubi, E.; Aramco, S.; Soyer, W., 2011. Sub-basalt imaging with broadband magnetotellurics in NW Saudi Arabia. SEG San Antonio 2011 Annual Meeting.

Constable S. C. et al. Occams' inversion: A practical algorithm for generating smooth models from electromagnetic sounding data. GEOPHYSICS, VOL. 52, NO. 3 (MARCH 1987): P 289-300,

Cordani U. G. et al. Estudo preeliminar de integração do pré-cambriano com os eventos tectônicos das bacias sedimentares brasileiras. Ciência Técnica Petróleo, p. 1 70, 1984.

Holz M.; Kalkreuth W.; Rolim S. B. A. 2010. Extension of the Parana' Basin to offshore Brazil: Implications for coalbed methane evaluation. Marine and Petroleum Geology, v. 27, issue 5.

Jones A.G. 1998. Static shift of magnetotelluric data and its removal in a sedimentary basin environment. Geophysics, v. 53, n. 7, p. 967-978, 1988.

Lima E. F.; Waichel B. L.; Rossetti L. M. M.; Viana A. R.; Scherer C. M.; Bueno G. V.; Dutra G. 2012. Morphologic and Petrographical Patterns of the pahoehoe and a'a' flows of Serra Geral Formation in the Torres Syncline (Rio 
Grande do Sul State, Brazil) Revista Brasileira de Geociências, v. 42(4), p. 744-753.

Lopes R. C. et al. Projeto a borda leste da bacia do paraná: integração geológica e avaliação econômica. Congresso Brasileiro de Geologia (34. : 1986 : Goiania, Go). Anais. Goiania : SBG, 1986.

Menezes P. T. L.; Fundamentos do Método Magnetotelúrico na Exploração de Hidrocarbonetos; Rio de Janeiro: SBGf, 2013. 208 pp.

Moritz, M.F., White, R.S., 2001. Seismic structure of basalt flows from surface seismic data, borehole measurements, and synthetic seismogram modelling. Geophysics 66 (6), 1925-1936

Philipp, R.P. ET al. Estruturação do arco de rio grande e da sinclinalde torres, bacia do Paraná, RS: evideências por levantamentos magnetotelúricos.

Renner L. C.; Hartmann L. A. 2010. Geoquímica de Sills Basálticos da formação Serra Geral, sul do Brasil, com base em rocha total e micro-análise de minerais.

Rodi, W., Mackie, R. L., 2001. Nonlinear conjugates gradients algorythm for 2-D magnetotelluric inversion, in press. Geophysics 66, 174-187.

Rosa, A., Tassini, J., Backus, M., 1982. Seismic data quality in a basalt covered basin. 62th SEG meeting, expanded abstracts, Society of Exploration Geophysicists (SEG), Tulsa, USA, S5.5.

Rossetti L. M. M.; Lima E. F. 2014. Arcabouço Estratigráfico da Formação Serra Geral no Vale Principal da Sinclinal de Torres.

Soares, A. V. Jr., Machado, F. B., Fernandes, M. A., Souza, I. A., Hasui, Y., Morales, N. 2012 Compartimentação estrutural do sinclinal de torres e espessura crustal da região entre os arcos de ponta grossa e rio grande a partir de gravimetria. In. $46^{\circ}$ Congresso Brasileiro de Geologia, resumo.

Vozoff, K., 1991. The magnetotelluric method. In: Nabighian, E. (Ed.), Electromagnetic methods in Applied Geophysics. Society of Exploration Geophysics, Tulsa, pp. 641-711.

Zevallos, I.; Assumpcao, M.; Padilha, A. L.. 2009. Inversion of teleseismic receiver function and magnetotelluric sounding to determine basement depth in the Parana Basin, SE Brazil. JOURNAL OF APPLIED GEOPHYSICS; v. 68, n. 2, p. 231-242, JUN 2009. 


\section{Anexos}

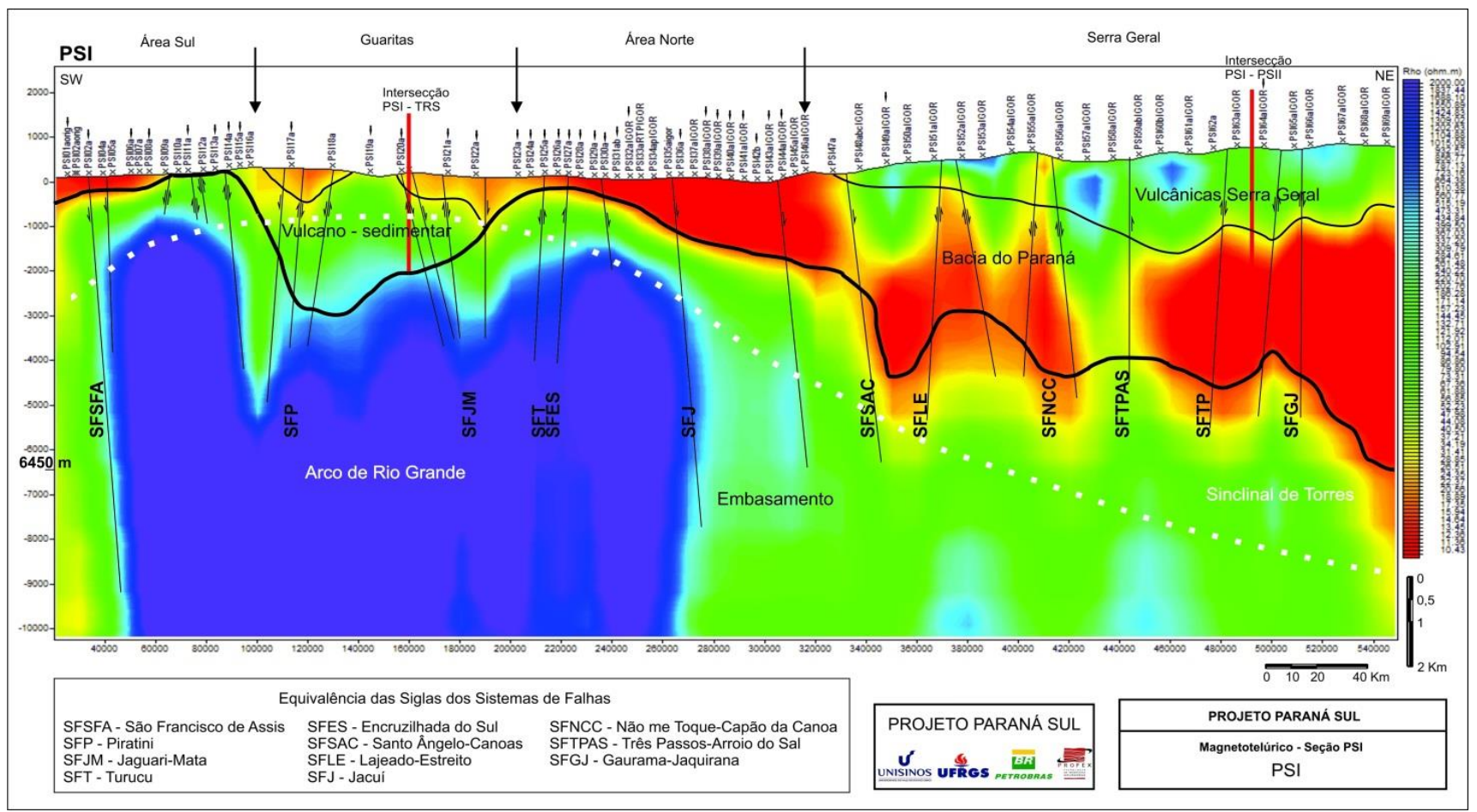

Figura 5 - Modelagem 2D da transecta PS1. Cores frias representam alto valor de resistividade e cores quentes baixo valor. A Sinclinal de Torres está representada pelo traço branco. As descontinuidades das linhas amarelas representam as falhas no pacote sedimentar (Modificado de Phillip et al 2014). 